\title{
Low-Frequency Tone Pips Elicit Exaggerated Startle Reflexes in C57BL/6J Mice with Hearing Loss
}

\author{
James R. Ison and Paul D. Allen \\ Department of Brain and Cognitive Sciences, Meliora Hall, University of Rochester, Rochester NY 14627, USA
}

Received: 4 September 2002; Accepted: 1 April 2003; Online publication: 6 June 2003

\section{ABSTRACT}

The strength of the acoustic startle reflex (ASR) as a function of age was studied in adult $\mathrm{C} 57 \mathrm{BL} / 6 \mathrm{~J}$ and CBA/CaJ mice, because altered ASR levels are a potential behavioral consequence of the neural reorganization that accompanies the early-onset hearing loss of the C57BL, in contrast to the normal-hearing CBA. For C57BL mice at 14-36 weeks of age, compared with 7-week-old mice, high-frequency thresholds measured with the auditory brainstem response (ABR) were less sensitive by about $25-30 \mathrm{~dB}$ while the hearing loss at low frequencies was $10-15 \mathrm{~dB}$, but by 60 weeks losses of $45-50 \mathrm{~dB}$ were present across the entire spectrum. Their ASR amplitudes for $16 \mathrm{kHz}$ tone pips were highest at 7 weeks and then declined with age, but, for $4 \mathrm{kHz}$ tones the ASR increased in strength at 18 weeks and beyond to levels above that of the younger mice. This hyperreactivity persisted even in 60-week-old mice. The ASR for $16 \mathrm{kHz}$ stimuli was positively correlated with hearing sensitivity, but the ASR for $4 \mathrm{kHz}$ stimuli was positively correlated with hearing loss for mice that were 18-36 weeks of age. Furthermore, ASR amplitudes for $4 \mathrm{kHz}$ stimuli were positively correlated with the $16 \mathrm{kHz}$ ASR in young C57BL mice but negatively correlated in older mice. There were no similar ASR or ABR changes in adult CBA mice through 19 weeks of age. Correlations between ASR and ABR scores were always weakly positive, and correlations between $4 \mathrm{kHz}$ and $16 \mathrm{kHz}$ ASR amplitudes were always strongly positive. The

Correspondence to: James R. Ison, Ph.D. • Department of Brain and Cognitive Sciences - Meliora Hall - University of Rochester - Rochester NY 14627 - Telephone: (585) 275-8461; fax: (585) 442-9216; email: Ison@bcs.rochester.edu
ASR data in older C57BL mice with hearing loss are consistent with reports describing their increased neural representation of low-frequency sounds and reinforce the value of this strain for studying the functional consequences that accompany age-related cochlear degeneration.

Keywords: aging, hearing loss, plasticity, startle

\section{INTRODUCTION}

Mice of the C57BL/6J strain exhibit a genetically determined progressive cochlear degeneration that begins at about 1-2 months of age in the basal cochlea and spreads throughout the entire organ of Corti over the course of the next 12-15 months. Its effects variously include fused and disorganized stereocilia, degenerating outer and inner hair cells and supporting cells including the spiral ligament, an atrophic stria vascularis, and loss in myelination and numbers of spiral ganglion cells (Mikaelian et al. 1974; Henry and Chole 1980; Shnerson et al. 1981; Willott et al. 1987; Li and Hultcrantz 1994; Hequembourg and Liberman 2001). These structural changes are more evident at the base compared with the cochlear apex. The resulting age-related decrement in auditory sensitivity has been demonstrated in increased absolute thresholds for auditory-evoked potentials and behavioral-psychophysical tasks (Mikaelian et al. 1974; Henry and Chole 1980; Shnerson and Pujol 1981; Willott 1986; Li and Borg 1991; Erway et al. 1993; Kazee et al. 1995; Spongr et al. 1997; Hequembourg and Liberman 2001). Although the 
specific details of the pattern of sensory loss across frequency vary across studies, the most common outcome is that the early loss is most serious for high tonal frequencies but then spreads across the spectrum of hearing with increasing age.

The focus of the present investigation concerns the consequences for behavioral responsivity to lowfrequency tonal stimuli of a change in threshold sensitivity that is biased toward higher frequencies. The presence of selective and progressive hearing loss in the C57BL mouse provides a unique methodology for examining neural plasticity in the central auditory system. Studying the central effects and behavioral consequences of their relatively slow progression of cochlear degeneration can complement the more commonly used methods to study neural plasticity, which use noise exposure, ototoxic drags, or physical trauma to degrade sensory input, as reviewed, e.g., by Harrison et al. (1996), Kaas (1996), Rajan and Irvine (1996), and Salvi et al. (1996).

A major physiological consequence of an acute frequency-limited loss of sensory input is a reorganization of central tonotopic representation. The auditory neurons that are located in tonotopic regions most sensitive to the lost frequencies are not silent in the absence of their normal input but come to respond with increased sensitivity to adjacent frequencies that are near the edge of residual hearing (Robertson and Irvine 1989; Harrison et al. 1991; Calford et al. 1993; Rajan et al. 1993; Eggermont and Komiya 2000). The more gradually developing cochlear degeneration that is the cause of hearing loss in the C57BL mouse has a similar effect (Willott 1996). The best frequencies of auditory neurons not only shift downward but also show an increase in their sensitivity to middle and low frequencies, with threshold levels near those previously obtained for high-frequency input. Their increased sensitivity is seen most strongly in auditory cortex (Willott et al. 1993), but is present also throughout the central nucleus of the inferior colliculus (Willott 1986) and, to a lesser extent, in the dorsal cochlear nucleus (Willott et al. 1991). Willott (1986) measured multiunit tuning curves in his mapping of the changing dorsoventral tonotopic organization of the inferior colliculus (IC) in C57BL mice from 1 to 12 months of age. He found downward shifts in best frequency extending over at least 1 octave in the normally high-frequency ventral regions of the IC in the older mice. Smaller upward shifts in best frequency were present in the formerly very-low-frequency dorsal edge of the inferior colliculus. (This may result from a greater loss in sensitivity for very low compared with middle frequencies, as shown by some but not all investigators.) In addition, the sensitivity of the lower tail of the neural tuning curves was increased by about $20-30 \mathrm{~dB}$ throughout the IC, save again for its most dorsal low-frequency extent.

One expected result of this downward shift of the best frequencies of central auditory neurons and of the greater sensitivity in the low-frequency tails of their tuning curves is that the auditory cortex and the IC, and possibly other structures including the dorsal cochlear nucleus, must become hypersensitive to lowand mid-frequency acoustic signals. It can be expected that central hyperexcitability would have significant sensory and behavioral consequences, and, in fact, Willott and his colleagues have demonstrated that low- or mid-frequency brief tones produce greater prepulse inhibition and facilitation of the acoustic startle reflex (ASR) when they are presented just prior to reflex elicitation, at ages for which highfrequency tones have become less effective (Willott et al. 1994; Willott and Carlson 1995; Willott 1996; Carlson and Willott 1996, 1998). Here we report on additional behavioral effects of hearing loss in the older C57BL mouse: We show that the vigor of the ASR elicited by low-frequency tone pips becomes exaggerated in these mice between about 2 and 4 months and that individual differences in this persistent hyperreflexivity are correlated with an increased severity of hearing loss. A subsidiary experiment showed that these behavioral effects are not obtained in the CBA strain of mice, which has no hearing loss at these ages.

\section{MATERIALS AND METHODS}

\section{Subjects}

The subjects in the main experiment were 29 C57BL/6J mice (13 male, 16 female). They began the experiment at $6-8$ weeks of age $(n=8$; mean age to closest week $=7), 18$ weeks $(n=8), 32-38$ weeks $(n=7$, mean age $=36$ weeks $)$, or $60-61$ weeks $(n=7$, mean age $=60$ weeks $)$. The subjects in the subsidiary experiment were $25 \mathrm{CBA} / \mathrm{CaJ}$ mice (11 male, 14 female). They were tested at 9-10 weeks of age $(n=9$, mean age $=10$ weeks $), 14$ weeks $(n=8)$, or 18-19 weeks (mean age $=19$ weeks). All mice were bred in the University of Rochester vivarium from parent stocks obtained from Jackson Laboratories (Bar Harbor, ME). They were group-housed in a constant climate and a 12/12 $\mathrm{h}$ normal L/D cycle and were tested in the daytime. Food and water were available ad libitum save during the test session. All procedures were approved by the University of Rochester Committee on Animal Resources and were in accord with the regulations of the Public Health Service and the Federal Animal Welfare Act. 


\section{Apparatus and procedures}

Details of the auditory brain stem response (ABR) testing apparatus and procedures have been published previously in Barsz et al. (2002). Briefly, binaural $\mathrm{ABR}$ audiograms were measured in response to tone pips of $3,6,12,24,32$, and $48 \mathrm{kHz}$ that were presented at the rate of 11 bursts/s with tone duration of $1 \mathrm{~ms}$, including $0.5 \mathrm{~ms}$ Blackman-windowed rise/fall times. The tone pips were presented through a leaf tweeter located at $0^{\circ}$ azimuth. Stimulus generation was controlled with a personal computer using a digital signal-processing platform (Tucker-Davis Technologies, Gainesville, FL, TDT AP2), and testing was conducted in a foam-lined IAC chamber (IAC, Bronx, $\mathrm{NY}$ ) in a quiet room adjoining the vivarium. Stimulus level was reduced by $10 \mathrm{~dB}$ SPL to below the threshold of the most prominent wave (typically the wave $4 / 5$ complex occurring at about $5 \mathrm{~ms}$ ) then increased in 3-5 dB steps to bracket the threshold.

Acoustic startle reflexes were measured to tone pips varying in frequency and in level. A mouse was confined for testing in an aluminum wire cage, oval in shape and $5 \mathrm{~cm}$ wide, $7 \mathrm{~cm}$ long, and $4 \mathrm{~cm}$ high. One mouse was tested at a time. The cage was mounted on a suspended acrylic platform to which an accelerometer was attached; it was placed within a sound-attenuating IAC room lined with sound-absorbing foam (inside dimensions approximately $2 \mathrm{~m} \times 2 \mathrm{~m} \times 2 \mathrm{~m}$; IAC). The force of the startle reflex was measured by the accelerometer located under the center of the cage, its signal amplified and integrated over a $100 \mathrm{~ms}$ period beginning with stimulus onset. The startle stimuli were tone pips, $70-120 \mathrm{~dB}$ (peak-to-peak, SPL linear scale), having a $20 \mathrm{~ms}$ duration, including $5 \mathrm{~ms}$ rise and fall times. The stimuli were provided by a realtime digital signal processor (TDT RP2) attenuated by a programmable attenuator (TDT PA5), then amplified and delivered to a Yamaha Professional Series Compression tweeter. The ambient noise level in the chamber was $<25 \mathrm{~dB}$ SPL for all frequencies above 125 Hz. Sound levels were measured with a 0.25-in. (6.4 mm) Bruel \& Kjaer microphone (Model 4135) and sound level meter (Model 2203) and an octave band filter (Model 1613). The speaker was mounted directly over the center of the cage at a distance of 15 $\mathrm{cm}$. The mouse was free to move in the cage but was typically aligned along its major axis, with its head at one end or the other. In calibrating the startle stimuli, the microphone was placed at the position of the mouse ear but with the cage removed from the apparatus. The measured stimulus levels at the positions of two ends of the cage were equal. The output from the accelerometer was amplified and routed to an A/ D converter (TDT RP2) and analyzed with custom software on a personal computer.

\section{Experimental design}

All of the startle conditions were given in a single test day. In the main experiment, a test consisted of 168 trials presented on average $20 \mathrm{~s}$ apart, with the session lasting about $1 \mathrm{~h}$. There were 28 stimulus conditions in all, consisting of tone pips given at 6 stimulus levels $(70,80,90,100,110$, and $120 \mathrm{~dB})$ at each of 4 frequencies $(4,8,16$, and $32 \mathrm{kHz})$, plus 4 no-stimulus baseline activity trials. All 28 conditions were given in random order within a block of trials, for a total of 6 blocks. Inspection of the stimuli subsequently revealed that the speaker distorted the $32 \mathrm{kHz}$ stimulus at high levels and, thus, the responses to this nominal frequency will not be reported. The procedure for CBA mice differed from that described above in that it did not include the $32 \mathrm{kHz}$ stimulus in the test battery; thus, a total of only 126 trials were given. The first two cohorts of C57BL mice received two ASR tests, 6-7 weeks apart, and the second two groups received only one test. One group was tested first at a mean of 7 weeks of age and again at 14 weeks, and the second group was tested first at 18 weeks and then again at 24 weeks. The third group of C57BL mice was tested at a mean of 36 weeks of age, and the fourth group was tested at a mean of 60 weeks. The ABR measures were collected for all mice in the appropriate groups at 14, 24, 36, and 60 weeks of age, with few exceptions within a week of the ASR test. In addition, 5 of the 8 mice in the first C57BL group received an ABR test at 7 weeks of age. The CBA mice were tested only once, at 10,14 , or 19 weeks of age, with the ABR measures given within a week of the behavioral test with few exceptions (one mouse in the 10-week-old group did not receive an ABR test).

The ASR and ABR means were subjected to mixeddesign analysis of variance (ANOVA) with age and sex as between-S variables. The within-S variables were frequency for the $\mathrm{ABR}$, and intensity and frequency for the ASR. Degrees of freedom were adjusted for nonhomogeneity of intercondition correlations by the Huynh-Feldt correction (Huynh and Feldt 1976).

\section{RESULTS}

Hearing thresholds

Figure lA shows the mean $( \pm$ SEM) ABR thresholds for the 5 ages at which ABRs were collected in the C57BL mice. The results of the overall ANOVA of these data are presented in Table 1, showing significant losses in threshold sensitivity with increased age that depended on the signal frequency. The initial loss of sensitivity between 7 and 14 weeks was present across the entire spectrum, though high frequencies were more affected than low (at $3 \mathrm{kHz}$, the mean $\pm \mathrm{SEM}=$ 

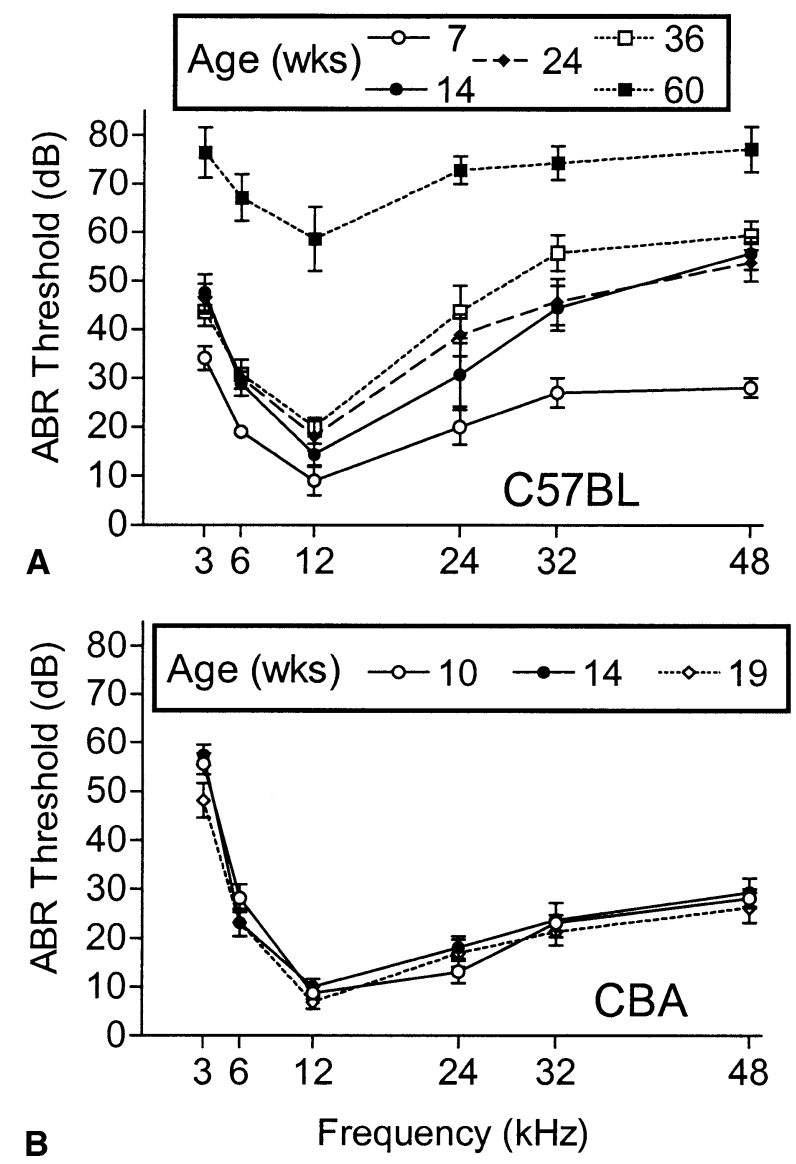

FIG. 1. Mean $A B R$ thresholds $( \pm S E M)$ for $C 57 B L / 6)$ mice $(A)$ and $\mathrm{CBA} / \mathrm{CaJ}$ mice $(\mathrm{B})$ with increasing age. The numbers of $\mathrm{C} 57 \mathrm{BL}$ mice at each age are $5,8,8,7$, and 7 , and of CBA mice, 8,8 , and 8 , from youngest to oldest. Note the (1) early hearing loss in the C57BL strain across the spectrum, but especially at high frequencies; (2) small additional loss of sensitivity across the next three age groups; and (3) final large and widespread loss in sensitivity in the oldest group. The CBA mice showed no hearing loss between 10 and 19 weeks of age.

$13.5 \pm 5.2 \mathrm{~dB}$, and at $48 \mathrm{kHz}, 27.6 \pm 4.6$; for the interaction between frequency and age, $p<0.01)$. The average progression of hearing loss between 14 and 36 weeks of age consisted of only a slight continuing increase in high-frequency thresholds, with the maximum single difference between these two extreme groups being just $13 \mathrm{~dB}( \pm 24 \mathrm{~dB}, p>0.50)$ obtained at $24 \mathrm{kHz}$. Compared to these middle-aged groups, the mice tested at 60 weeks of age sustained a major loss of sensitivity across the spectrum, especially in the middle to low frequencies that had remained relatively constant in the middle-aged mice. The loss of threshold sensitivity between the ages of 36 and 60 weeks ranged from $38.6( \pm 6.9) \mathrm{dB}$ at $12 \mathrm{kHz}$ to 17.8 $( \pm 5.6 \mathrm{~dB})$ at $48 \mathrm{kHz}$. The loss at $4 \mathrm{kHz}$ was $32.9( \pm$ 6.0) dB.

Although the pattern of hearing loss across frequency differed between the age groups, hearing losses across frequencies within an age group were highly correlated so that, e.g., mice with poorer hearing for high frequencies tended also to have poor low-frequency hearing for their age. The average correlation between the ABR thresholds at 3 and $48 \mathrm{kHz}$ across the age grouping from 14 to 36 weeks was calculated from Fisher's $r$ to $Z$ transformation, to yield $r=+0.70, d f=20, p<0.01$. Although the hearing thresholds for these two frequencies were correlated across animals in this age range, by 36 weeks of age the high-frequency hearing loss at $48 \mathrm{kHz}$ (mean $=28.1, \mathrm{SD}=9.4$ ) was greater than that obtained at $3 \mathrm{kHz}($ mean $=11.9, \mathrm{SD}=9.0)$, in comparison to thresholds obtained at 7 weeks of age. The mean difference between the severity of the hearing loss for these two frequencies was $16.2 \mathrm{~dB}(\mathrm{SD}=8.6)$, $t(22)=9.05, p<0.01$.

Figure 1B shows the ABR thresholds for the CBA mice at 10,14 , and 19 weeks of age, which were stable over this period (see Table 1). The thresholds of these CBA mice approximated those of the 7-weekold C57BL mice, save for their being less sensitive at 3 $\mathrm{kHz}(p<0.01)$.

\section{Startle behavior}

Figure $2 \mathrm{~A}-\mathrm{C}$ shows the mean ASR amplitudes $( \pm \mathrm{SEM})$ in each test for each C57BL cohort across all six levels for the 4,8 , and the $16 \mathrm{kHz}$ stimuli. Most prominent in these data is the increase between 14 and 18 weeks of age in the amplitude of the startle reflex elicited by the $4 \mathrm{kHz}$ tone pip. This enhancement of the lowfrequency ASR persisted even in the oldest group of mice. A similar though less pronounced effect was evident at the lower levels of the $8 \mathrm{kHz}$ ASR, while, in contrast, the ASR for the $16 \mathrm{kHz}$ stimulus declined with increasing age. The results of the overall ANOVA of these data are provided in Table 1, showing significant effects for the main effect of age and the interactions of age with signal frequency and signal intensity. Separate ANOVA grouping across different ages at both 4 and $8 \mathrm{kHz}$ showed that the recovery in the response between the 14 -week and the 18-week age groups was significant $(p<0.01)$. The 18-week-old age group also responded more vigorously than the 7 week-old group at $4 \mathrm{kHz}(p<0.01)$, but there were no further significant increases with advancing age for this frequency $(p>0.1)$. At $8 \mathrm{kHz}$ the ASR fell between the adjacent 24-week and the 36-week age groups $(p<0.02)$. At $16 \mathrm{kHz}$ the ASR progressively but slowly declined with age $(p=0.057)$, and the ASR of the youngest group was significantly greater than that of the two oldest groups $(p<0.05)$.

The startle data for the CBA mice are shown in the 2D-F. The CBA mice had a higher asymptotic level of responsivity than the $\mathrm{C} 57 \mathrm{BL}$, though the ASR thresholds were comparable to those of the very 


\section{TABLE 1}

Major ANOVA results

\begin{tabular}{|c|c|c|c|}
\hline Source & ABR threshold & ASR amplitude & ASR threshold \\
\hline \multicolumn{4}{|l|}{ C57BL mice } \\
\hline Age & $F(4 / 29)=30.51^{* *}$ & $F(5 / 29)=4.01^{* *}$ & $F(5 / 40)=2.75^{*}$ \\
\hline Frequency & $F(5 / 145)=72.31^{* *}$ & $F(2 / 80)=59.28^{* *}$ & $F(2 / 80)=25.48^{* *}$ \\
\hline$F \times A$ & $F(20 / 145)=2.71^{* *}$ & $F(10 / 80)=6.62^{* *}$ & $F(10 / 80)=2.29^{*}$ \\
\hline Intensity & & $F(5 / 80)=75.52^{* *}$ & \\
\hline$I \times A$ & & $F(25 / 200)=3.10^{* *}$ & \\
\hline$F \times I$ & & $F(10 / 50)=42.20^{* *}$ & \\
\hline$F \times I \times A$ & & $F(50 / 400)=3.42^{* *}$ & \\
\hline \multicolumn{4}{|l|}{ CBA mice } \\
\hline Age & $F(2 / 21)<1$ & $F(2 / 22)<1$ & $F(2 / 22)<1$ \\
\hline Frequency & $F(5 / 100)=181.68^{* *}$ & $F(2 / 44)=59.08^{* *}$ & $F(2 / 44)=454.63^{* *}$ \\
\hline$F \times A$ & $F(10 / 100)=1.24$ & $F(4 / 44)<1$ & $F(4 / 44)=2.72^{*}$ \\
\hline Intensity & & $F(5 / 110)=49.03^{* *}$ & \\
\hline$I \times A$ & & $F(10 / 110)<1$ & \\
\hline$F \times I$ & & $F(10 / 220)=21.77^{* *}$ & \\
\hline$F \times I \times A$ & & $F(20 / 220)<1$ & \\
\hline
\end{tabular}

${ }^{*} p<0.05 ;{ }^{* *} p<0.01$

young C57BL prior to the onset of hearing loss (see below). A striking difference in literal "jumpiness" between the two strains is always very apparent in handling the mice in their home cages and, thus, it is not specifically an acoustic reflex phenomenon. Overall, the youngest group was the most reactive of the CBA mice for each of the stimulus frequencies, but there were no significant differences between the three age groups (see Table 1). Of particular interest in the comparison between strains is the observation for the $4 \mathrm{kHz}$ stimulus that, although at $120 \mathrm{~dB}$ the CBA mice responded more than the C57BL $[\mathrm{F}(1 /$ $51)=18.93, p<0.01]$, for lower stimulus intensities, the $\mathrm{C} 57 \mathrm{BL}$ that were at least 18 weeks of age were more responsive that the CBA mice $[\mathrm{F}(1 / 51)=12.72$, $p<0.01]$.

Figure 3A shows the mean ASR thresholds for the C57BL mice as a function of age and frequency, with the threshold being determined as the lowest level for each mouse at which the ASR was significantly greater than its activity score in a $t$-test with $\alpha=0.05$, one-tail. If the ASR was not significantly different from activity at any intensity, then an arbitrary threshold score of $130 \mathrm{~dB}$ was recorded. An overall decrement in threshold sensitivity with increased age was most apparent for the two oldest groups with the $16 \mathrm{kHz}$ stimulus, while in contrast the thresholds for the 4 $\mathrm{kHz}$ stimulus showed a slight increase in sensitivity with age. There was a relatively large increase in threshold from 7 weeks to 14 weeks of age for the 8 and $16 \mathrm{kHz}$ stimuli; it was then reversed at 18 and 24 weeks. The overall ANOVA of these threshold data provided in Table 1 showed significant effects for both age and frequency and for their interaction. The
ANOVA at separate frequencies provided a significant effect of age for only the $16 \mathrm{kHz}$ stimulus $(p<0.01)$, but at $8 \mathrm{kHz}$ there was a significant increase in the ASR threshold from 7 to 14 weeks of age $(p<0.01)$; then the threshold recovered at 18 weeks of age $(p<$ 0.01). The ASR thresholds for the CBA mice were relatively stable over the early period of 10-19 weeks, except for a small but significant $(p=0.04)$ increase in the threshold for the $4 \mathrm{kHz}$ stimulus in the oldest group. Comparisons of the two strains between 7 and 19 weeks of age resulted in a significant interaction between strain and frequency $[F(2 / 86)=29.01, p<$ $0.01]$, with the CBA mice having lower thresholds for the $16 \mathrm{kHz}$ stimulus $[F(1 / 43)=26.63, p<0.01]$ but higher thresholds for the $4 \mathrm{kHz}$ stimulus $[F(1 /$ 43) $=12.92, p<0.01]$.

The observation that for the C57BL mice the mean responding to the $16 \mathrm{kHz}$ stimulus declined while the response to the $4 \mathrm{kHz}$ stimulus increased with age prompted an examination of the correlation across these mice within each age group between their 4 $\mathrm{kHz}$ ASR and their $16 \mathrm{kHz}$ ASR. For this set of analyses the maximum mean response at any level for the $4 \mathrm{kHz}$ stimulus for each mouse was paired with its maximum mean response at any level for the $16 \mathrm{kHz}$ stimulus. For the 7- and 14-week ages in the C57BL mice, the amplitudes of these two responses were positively correlated, $r=+0.87$ and $r=+0.77$. These values are not independent but were provided by the same 8 subjects tested at both times. Their average was calculated using Fisher's $r$ to $Z$ transformation to yield a single significant positive correlation, $r=+0.83, d f=7, p<0.01$. The correlations were also positive in the CBA mice, at $r=+0.92,+0.93$, and 

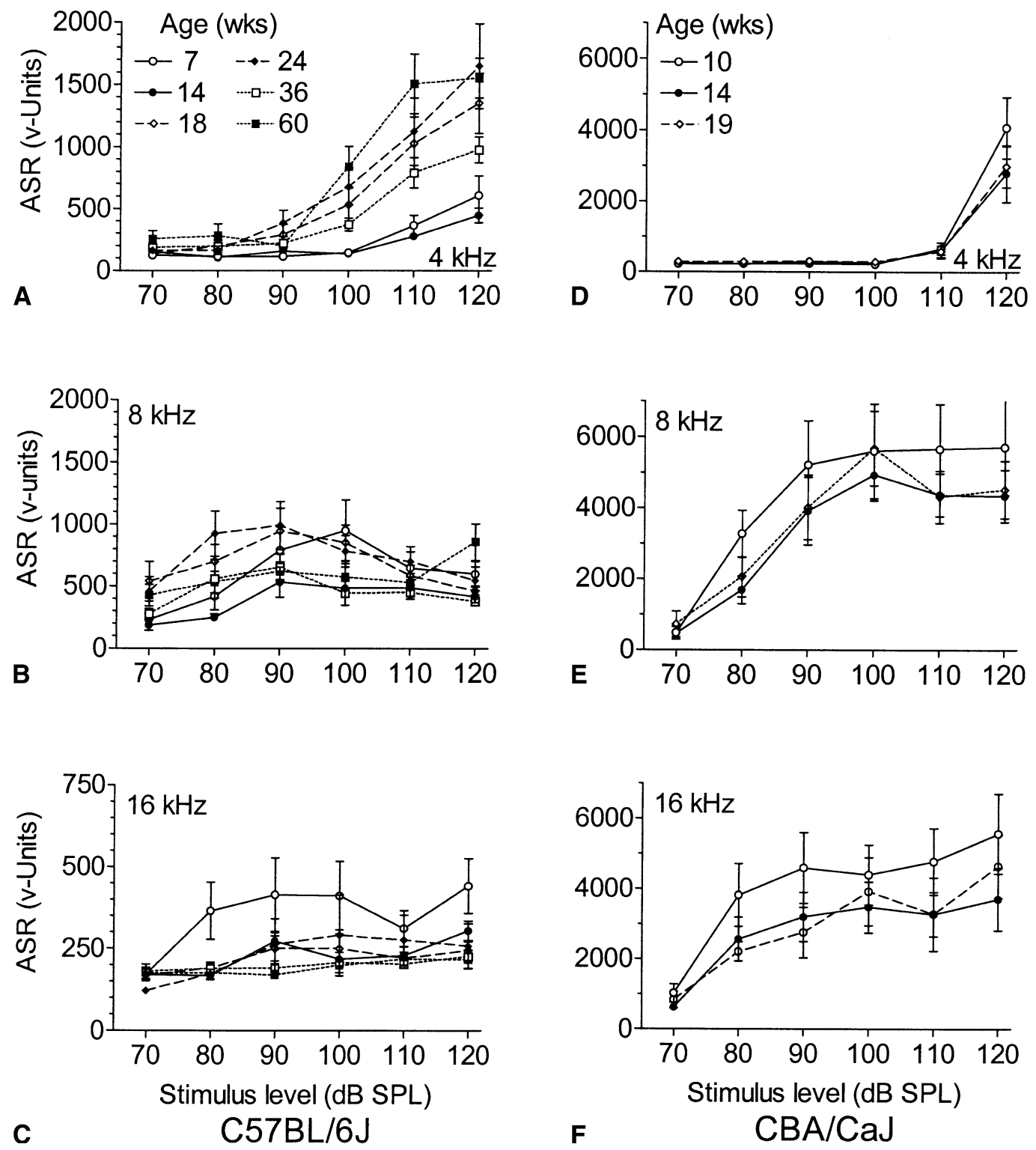

FIG. 2. Mean ASR amplitudes ( \pm SEM) across frequency and level, (A-C) for six ages of C57BL mice, 7-60 weeks, (D-F) for three ages of CBA mice, 10, 14, and 19 weeks. The ASR is measured in arbitrary voltage units, a linear function of the force exerted on the accelerometer by the startle flinch. In the C57BL mice, note the (1) overall

$+0.78(p<0.01,0.01,0.05)$ for the 10-, 14-, and 19week-old mice, respectively. In sharp contrast, for the C57BL mice at 18 and 24 weeks of age, the amplitudes of the two responses were negatively correlated, $r=-0.61$ and $r=-0.72$. Averaging these two numbers provided by the 8 subjects in this group yielded a single significant negative correlation, $r=-0.67$, $d f=7, p<0.05$. For the 36 -week- and the 60 -week-old C57BL groups, the correlations remained negative but were not significant $(r=-0.32, \quad r=-0.34$, $d f=6,6)$.
Relationship between ABR thresholds and ASR strength

Figure 4 presents three scatterplots showing the relationships between the ABR thresholds and ASR responses across all of the C57BL mice. The ABR values are the mean thresholds for all of the frequencies combined, 3-48 kHz, as there was no apparent difference between mice in the pattern of the progression of hearing loss across frequency (the overall mean ABR correlated with each separate frequency, 

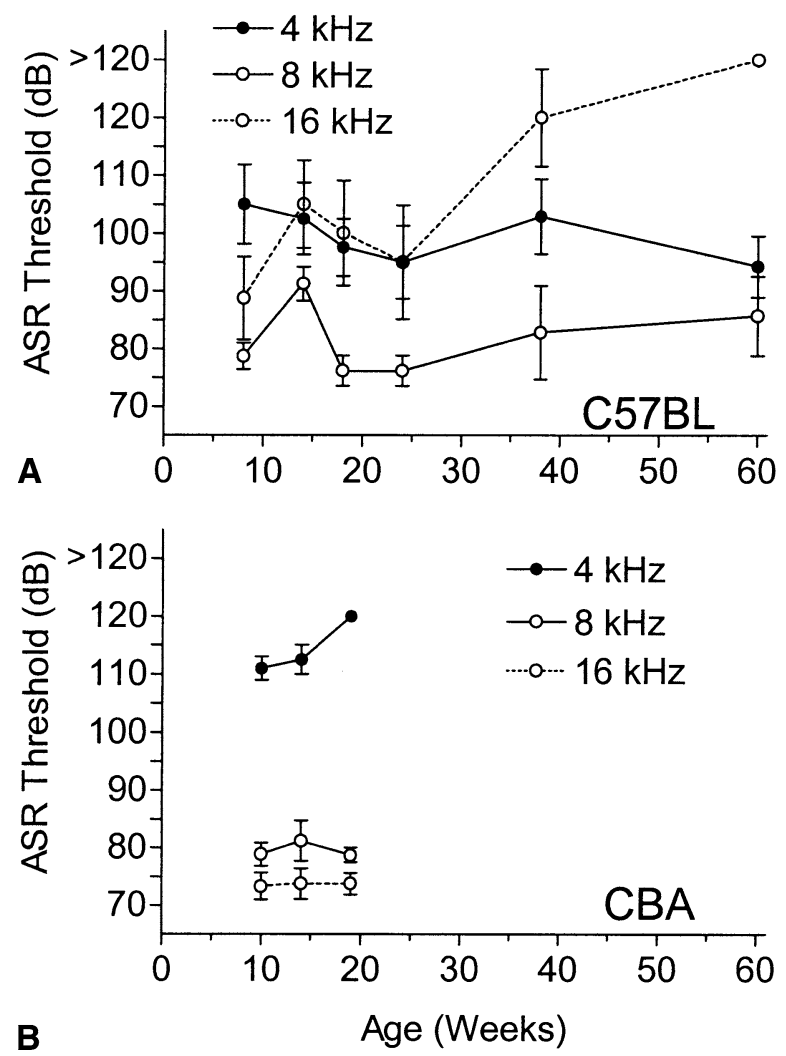

FIG. 3. Mean ASR thresholds $( \pm$ SEM) as a function of age and stimulus frequency in C57BL (A) and CBA mice (B). The threshold was the lowest level at which the ASR differed $(p<0.05$, one-tail) from the activity baseline. In the C57BL mice, the $16 \mathrm{kHz}$ ASR threshold increased with age, except for a slight drop or plateau from 14 to 24 weeks of age. The only other significant effects of age were for $8 \mathrm{kHz}$, for the loss of sensitivity at 14 weeks followed by a recovery at 18 and 20 weeks of age. There were no threshold changes in the CBA mice. Compared with the CBA mice, the C57BL mice had lower thresholds for the $4 \mathrm{kHz}$ stimuli but higher thresholds for the $16 \mathrm{kHz}$ stimuli.

$+0.88<r<+0.91)$. The ASR values are the maximum mean response amplitude at any level for each ASR frequency. The linear regression lines for each group are displayed in Figure 4, and Table 2 provides the Pearson $r$ correlation coefficients for each of the groups at each frequency. The sample sizes were generally too small for single $r$ values to reach conventional levels of significance, except for the negative relationship between the ABR threshold and 16 $\mathrm{kHz}$ ASR data in the 38-week-old group $(p<0.05)$. However, it may also be noted that save for one outlier in the 24-week-old mice, the four regression lines for the $16 \mathrm{kHz}$ stimulus were approximately collinear. An overall mean correlation was calculated for the 4 groups by using Fisher's $r$ to $Z$ transformation. This calculation yielded a mean of $r=-0.48(d f=26, p<$ 0.01 ), indicating that overall the maximum amplitude of the ASR to the $16 \mathrm{kHz}$ stimulus was more vigorous in the mice in each group with greater hearing sen-
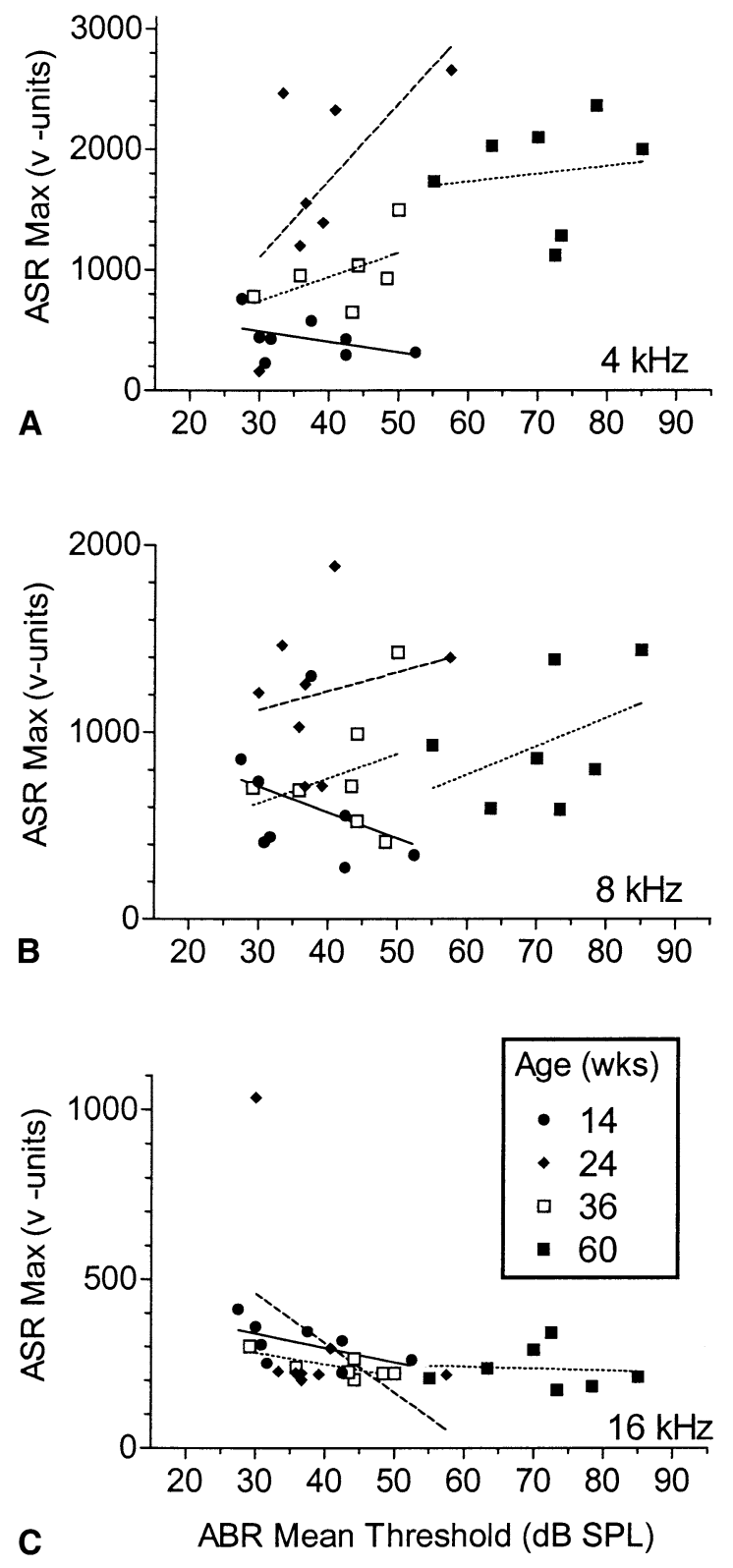

FIG. 4. Scatterplots showing the relationship for the C57BL mice between the mean ABR threshold (across all frequencies) and the maximum mean ASR value at any level. Each point represents the data from one mouse, and the mice appear once for each of the three ASR frequencies. Note the negative slope of the regression lines at 16 $\mathrm{kHz}$ for all ages and for all the ASR frequencies at 14 weeks of age, these showing that higher ASR values accompanied greater hearing sensitivity. All other slopes were positive, showing the tendency for the mice with greater hearing loss to respond more vigorously tolow-frequency stimuli within the older groups. (Pearson productmoment correlation coefficients are provided in Table 2.)

sitivity. The direction of this relationship in the 14week-old group was the same for each ASR frequency, but the pattern was reversed in the older groups at both 8 and $4 \mathrm{kHz}$. At these frequencies and for these age groups, the C57BL mice with greater hearing loss tended to respond more vigorously. This correlation was not significant for $8 \mathrm{kHz}$, but for the $4 \mathrm{kHz}$ sti- 


\section{TABLE 2}

ASR and ABR correlations across age in C57BL mice

\begin{tabular}{lccl}
\hline Age $(w k)$ & ABR and $4 \mathrm{kHz}$ & $8 \mathrm{kHz}$ & $16 \mathrm{kHz}$ \\
\hline 14 & $r=-0.43$ & -0.35 & -0.58 \\
24 & $r=+0.65$ & +0.21 & -0.42 \\
36 & $r=+0.55$ & +0.28 & -0.76 \\
60 & $r=+0.14$ & +0.43 & -0.09 \\
Mean: & $24-60 \mathrm{wk}$ & & $14-60 \mathrm{wk}$ \\
& $r=+0.47^{*}$ & & $-0.48^{* *}$ \\
\hline
\end{tabular}

$* p<0.05 ; * * p<0.01$.

mulus the average correlation of $r=+0.47$ between the mean ABR threshold and the maximum ASR was significant $(d f=19, p<0.05)$. For the CBA mice, the correlations between the overall ABR mean and the maximum ASR values were positive for each frequency $(r=+0.19,+0.16,+0.30$ for 4,8 , and $16 \mathrm{kHz}$, respectively), but none was significantly different from a null effect $(p>0.1)$

\section{DISCUSSION}

The primary outcome of this experiment was that hearing loss in the older C57BL mouse is accompanied by an increase in the strength of the acoustic startle reflex when it is elicited by low-frequency tone pips, particularly at $4 \mathrm{kHz}$, while its elicitation by highfrequency stimuli $(16 \mathrm{kHz})$ is diminished. Similar findings have been reported showing an increase in the reflex-modifying effects of tone pips: Low- and mid-frequency tones provide greater prepulse inhibition in old C57BL mice compared with the young, while high-frequency tones provide less prepulse inhibition (Willott et al. 1994; Willott and Carlson 1995; Carlson and Willott 19961998). Reflex strength at 4 $\mathrm{kHz}$ varied across C57BL mice within each group in the present experiment. These individual differences in the ASR were correlated with the severity of their hearing loss as indicated by ABR thresholds. In contrast, the strength of the ASR elicited by high-frequency tones was correlated with their residual hearing sensitivity. This association between hearing loss and ASR vigor for low-frequency eliciting stimuli suggests that the progress of hearing loss and the increasing diminution of afferent input to the central auditory system is the cause of the hyperreflexia. Also consistent with this idea is the observation that within the groups of older mice the strength of the ASR to the $4 \mathrm{kHz}$ stimulus was negatively correlated with the ASR to the $16 \mathrm{kHz}$ stimulus. This finding suggests that these two effects have a common cause in individual differences in hearing loss. A related finding of con- siderable interest is that of Willott et al. (1993), who reported less tonotopic reorganization in the auditory cortex of several older mice that had relatively better high-frequency hearing as measured in the ABR. In the present experiment, the pattern of correlations between the ASR and the ABR did not vary across ABR frequency; however, because the severity of hearing loss at $48 \mathrm{kHz}$ was more than twice that obtained at $3 \mathrm{kHz}$ in the middle age groups, it seems most reasonable to conclude that these individual differences in behavior result because of the differences in high-frequency hearing loss. None of these changes in reflex strength developed in CBA mice, a strain that does not develop peripheral hearing loss at comparable ages.

The apparently delayed emergence of reflex enhancement compared to the development of hearing loss is of further theoretical importance. The youngest group of mice had demonstrated a significant hearing loss in the seven-week period intervening between the first and second ASR tests, but the group had a decrement in reflex strength rather than exaggeration. After their initial hearing loss the thresholds in C57BL mice appeared to stabilize, as the ABRs recorded at 14 weeks overlapped those obtained at 24 and at 36 weeks of age. [It may be noted that this uneven progression of hearing loss with age is similar to that reported for the $\mathrm{C} 57 \mathrm{BL}$ by $\mathrm{Li}$ and Borg (1991).] In contrast to the loss of reflex strength at 14 weeks, the mice tested at 18 and at 24 weeks of age showed a significant recovery of the ASR threshold at $8 \mathrm{kHz}$ and a significant increase in ASR amplitudes at $4 \mathrm{kHz}$. Furthermore, while at 14 weeks of age the ASR maxima at 4 and at $16 \mathrm{kHz}$ were positively correlated, by 18 and 24 weeks of age the correlation for these two frequencies was negative. Thus, both the strength of the low-frequency ASR and its pattern of correlations with the ABR and the highfrequency ASR changed over this period of time, though the degree of hearing loss as indexed by the $A B R$ values was relatively stable. Assuming that ABR threshold shifts provide an appropriate metric for gauging changes in acoustic input to the central auditory system, the increase in the vigor of the lowfrequency ASR would appear to be a consequence of the increasing duration rather than increasing severity of hearing loss, and, presumably, this evolution of startle enhancement correlates with a progression of some form of neuronal reorganization resulting from deficits in high-frequency input.

The enhanced amplitudes and lower thresholds of auditory-evoked potentials that result because of noise trauma have been hypothesized to result from its greater effect on inhibitory rather than excitatory processes, this yielding an abnormal imbalance in favor of excitation in the central auditory system 
(Salvi et al. 1996). A similar argument may be appropriate for understanding the effects of hearing loss on ASR amplitudes and thresholds. A useful role for inhibition in moderating the normal ASR is suggested by the idea that the relatively intense low-frequency stimuli used in ASR experiments must activate a considerable extent of the basilar membrane and the auditory nerve. Thus, these stimuli have the potential to engage a high proportion of the middleand high-frequency regions in the central auditory system. For the mouse with normal hearing, the CBA mouse, for example, or the very young C57BL, we assume that the intrinsic inhibitory sidebands and the feedback and feedforward loops of the central auditory nervous system would normally serve to limit this otherwise expansive excitatory effect of intense lowfrequency stimuli. It then seems reasonable to propose, following the thoughts of Salvi et al. (1996) as well as Szczepaniak and Møller (1996), that the older C57BL mouse with high-frequency hearing loss has lost these inhibitory processes that normally serve to restrain the excitatory influence of low-frequency sounds. The observation that an exaggerated ASR takes some time to appear suggests that this inhibitory deficit is slow to develop following degenerative hearing loss, in contrast to the near immediate effect of noise trauma. This could be interpreted as either a gradual loss of inhibitory function, thus unmasking already present excitatory connections, or perhaps reflecting the growth of excitatory connections providing a new low-frequency input to high-frequency neurons that are not subject to normal inhibition.

There is one further complication in the startle literature for C57BL mice: Startle stimuli that contain power at high as well as low frequencies do not produce an exaggerated ASR. The ASR elicited by wideband noise stimuli is diminished with hearing loss in the C57BL mouse (Ison et al. 1998), as is the ASR elicited by brief $(10 \mathrm{~ms})$ low-frequency tone pips with very brief $(1 \mathrm{~ms})$ rise and fall times that provide a transient click at their onset (Parham and Willott 1988). This apparent difference in the effects of hearing loss on the response to relatively pure sinusoidal startle stimuli versus noise bursts or clicks especially needs further research and theoretical elaboration. One potentially important difference between the two conditions may be the extent to which high-intensity, high-frequency components of a wideband noise startle stimulus remain able to stimulate the high-frequency regions of the cochlea and the lower brain stem and, hence, use the normal afferent pathways to activate the more rostral high-frequency regions. Age-related tonotopic reorganization is absent in the ventral cochlear nucleus and minimal in the dorsal cochlear nucleus (Willott et al. 1991), and the high-frequency regions at these sites lose threshold sensitivity without being taken over by lowfrequency input. Their residual sensitivity to high-intensity stimulation may be sufficient to activate the normal suppressive pathways hypothesized to limit the spread of excitation from low- to high-frequency areas at more rostral sites in the auditory brain stem, in contrast to the failure of inhibition when those regions are abnormally activated by low-frequency input.

The progressive pattern of high-frequency hearing loss in the middle-aged C57BL mouse resembles that of many humans with either age-related sensoryneural hearing loss or noise-induced hearing loss. For example, the changes in human thresholds across different age groups (Brant and Fozard 1990) show high-frequency hearing loss beginning prior to 30 years of age, which is similar to the hearing loss observed in the adult C57BL mouse, with not unreasonable adjustment for the early maturation and short life span of mice. It seems unlikely that the effects of progressive hearing loss that we report here for the C57BL mouse are restricted to this specific type of cochlear pathology, especially because the effects are phenomenologically similar to those of different forms of acoustic trauma seen in other animal models. Analogous signs of hyperexcitability may be demonstrable in other species, including humans, that have progressive frequency-specific hearing loss. The present results reinforce the usefulness of this murine model of hearing loss and suggest the possibility that the robust form of hyperreflexia seen in the hearing-impaired C57BL mouse might be valuable as a target for testing potential therapeutic interventions for other hearing problems that involve abnormal loudness perception (Phillips and Carr 1998).

\section{ACKNOWLEDGMENTS}

The work reported here was supported by Grant AG095247 from the National Institute on Aging of the NIH. We are grateful to John Housel for collecting the ABR measures. A report of a preliminary experiment with similar results was presented at the Meetings of the Association for Research in Otolaryngology, 2000, and the present data on the C57BL mice were presented at the Meeting of the Society for Neuroscience, 2001.

\section{REFERENCES}

Barsz K, Ison JR, Snell KB, Walton JP. Behavioral and neural measures of auditory temporal acuity in aging humans and mice. Neurobiol. Aging 23:565-578, 2002.

BRANT LJ, FozARD JL. Age changes in pure-tone hearing thresholds in a longitudinal study of normal human aging. J. Acoust. Soc. Am. 88:813-820, 1990. 
CALford MB, Rajan R, IRvine DR. Rapid changes in the frequency tuning of neurons in the cat auditory cortex resulting from pure-tone-induced temporary threshold shift. Neuroscience 55:953-964, 1993.

Carlson S, Willott JF. The behavioral salience of tones as indicated by prepulse inhibition of the startle response: relationship to hearing loss and central neural plasticity in C57BL/6J mice. Hear. Res. 99:168-175, 1996.

Carlson S, Willott J. Caudal pontine reticular formation of C57BL/6J mice: responses to startle stimuli, inhibition by tones, and plasticity. J. Neurophys. 79:2603-2614, 1998.

EgGermont JJ, Komiya H. Moderate noise trauma in juvenile cats results in profound cortical topographic map changes in adulthood. Hear. Res. 142:89-101, 2000.

Erway LC, Willott JF, Archer JR, Harrison DE. Genetics of agerelated hearing loss in mice: I. Inbred and F1 hybrid strains. Hear. Res. 65:125-132, 1993.

Harrison RV, Nagasawa A, Smith DW, Stanton SG, Mount RJ. Reorganization of auditory cortex after neonatal high frequency cochlear hearing loss. Hear. Res. 54:11-19, 1991.

Harrison RV, Ibrahim D, Stanton SG, Mount RJ. Reorganization of frequency maps in chinchilla auditory midbrain after long-term basal cochlear lesions induced at birth. In: Salvi RJ, Henderson D, Fiorino F, Colletti V (Eds.) Auditory System Plasticity and Regeneration. Thieme Medical Publishers, New York, pp 238$255,1996$.

Henry KR, ChOle RA. Genotypic differences in behavioral, physiological and anatomical expressions of age-related hearing loss in the laboratory mouse. Audiology 5:369-383, 1980.

Hequembourg S, Liberman MC. Spiral ligament pathology: a major aspect of age-related cochlear degeneration in C57BL/6 mice. J. Assoc. Res. Otolaryngol. 2:118-129, 2001.

Huynh H, FeldT LS. Estimation of the Box correction for degrees of freedom from sample data in randomized block and split-plot designs. J. Educ. Stat. 1:69-82, 1976.

Ison JR, Agrawal P, PAK J, Vaughn WJ. Changes in temporal acuity with age and with hearing impairment in the mouse: a study of the acoustic startle reflex and its inhibition by brief decrements in noise level. J. Acoust. Soc. Am. 104:696-704, 1998.

KAAS JH. Plasticity of sensory representations in the auditory and other systems of adult animals. In: Salvi RJ, Henderson D, Fiorino F, Colletti V (Eds.) Auditory System Plasticity and Regeneration. Thieme Medical Publishers, New York, pp 213-223, 1996.

Kazee AM, Han Ly, Spongr VP, Walton JP, Salvi RJ, Flood DG. Synaptic loss in the central nucleus of the inferior colliculus correlates with sensorineural hearing loss in the C57BL/6 mouse model of presbycusis. Hear. Res. 89:109-120, 1995.

LI HS, Borg E. Age-related loss of auditory sensitivity in two mouse genotypes. Acta Otolaryngol. 111:827-834, 1991.

Li HS, Hultcrantz M. Age-related degeneration of the organ of Corti in two genotypes of mice ORL. J. Otolaryngol. Relat. Spec. 56:61-67, 1994.

Mikaelian DO, Warfield D, Norris BA. Genetic progressive hearing loss in the C57/b16 mouse: Relation of behavioral responses to cochlear pathology. Acta Otolaryngol. 77:327-334, 1974.
PARHAM K, Willott JF. Acoustic startle response in young and aging C57BL/6J and CBA/J mice. Behav. Neurosci. 102:881-886, 1988.

Phillips DP, Carr MM. Disturbances of loudness perception. J. Am. Acad. Audiol. 9:371-379, 1998.

Rajan R, Irvine DRF. Features of, and boundary conditions for, lesion induced reorganization of adult auditory cortical maps. In: Salvi RJ, Henderson D, Fiorino F, Colletti V (Eds.) Auditory System Plasticity and Regeneration. Thieme Medical Publishers, New York, pp 224-237, 1996.

Rajan R, Irvine DR, Wise LZ, Heil P. Effect of unilateral partial cochlear lesions in adult cats on the representation of lesioned and unlesioned cochleas in primary auditory cortex. J. Comp. Neurol. 338:17-49, 1993.

Robertson D, Irvine DRF. Plasticity of frequency organization in auditory cortex of guinea pigs with partial unilateral deafness. J. Comp. Neurol. 282:456-471, 1989.

SAlvi RJ, WANG J, Powers N. Rapid functional reorganization in the inferior colliculus and cochlear nucleus after acute cochlear damage. In: Salvi RJ, Henderson D, Fiorino F, Colletti V (Eds.) Auditory System Plasticity and Regeneration. Thieme Medical Publishers, New York, pp 275-296, 1996.

Shnerson A, Pujol R. Age-related changes in the C57BL/6J mouse cochlea. I. Physiological findings. Brain Res. 254:65-75, 1981.

Shnerson A, Devigne C, Pujol R. Age-related changes in the C57BL/6J mouse cochlea. II. Ultrastructural findings. Brain Res. 254:77-88, 1981.

SPONGR VP, FloOd DG, Frisina RD, SALVI RJ. Quantitative measures of hair cell loss in CBA and C57BL/6 mice throughout their life spans. J. Acoust. Soc. Am. 101:3546-3553, 1997.

SZCZEPANIAK WS, MøLLER AR. Evidence of neuronal plasticity within the inferior colliculus after noise exposure: a study of evoked potentials in the rat. Electroencephalogr. Clin. Neurophys. 100:158-164, 1996.

WiLlotT JF. Effects of aging, hearing loss, and anatomical location on thresholds of inferior colliculus neurons in C57BL/6 and CBA mice. J. Neurophys. 56:391-408, 1986.

Willott JF. Auditory system plasticity in the adult C57BL/6J mouse. In: Salvi RJ, Henderson D, Fiorino F, Colletti V (Eds.) Auditory System Plasticity and Regeneration. Thieme Medical Publishers, New York, pp 297-316, 1996.

Willott JF, CarLsOn S. Modification of the acoustic startle response in hearing-impaired $\mathrm{C} 57 \mathrm{BL} / 6 \mathrm{~J}$ mice: prepulse augmentation and prolongation of prepulse inhibition. Behav. Neurosci. 109:396-403, 1995.

Willott JF, Jackson LM, Hunter KP. Morphometric study of the anteroventral cochlear nucleus of two mouse models of presbycusis. J. Comp. Neurol. 260:472-480, 1987.

Willott JF, Parham K, Hunter KP. Comparison of the auditory sensitivity of neurons in the cochlear nucleus and inferior colliculus of young and aging $\mathrm{C} 57 \mathrm{BL} / 6 \mathrm{~J}$ and $\mathrm{CBA} / \mathrm{J}$ mice. Hear. Res. 53:78-94, 1991.

Willott JF, Aitkin LM, McFadden SL. Plasticity of auditory cortex associated with sensorineural hearing loss in adult C57BL/6J mice. J. Comp. Neurol. 329:402-411, 1993.

Willott JF, Carlson S, Chen H. Prepulse inhibition of the startle response in mice: relationship to hearing loss and auditory system plasticity. Behav. Neurosci. 108:703-713, 1994. 